\title{
Influence of Couple Stresses and Thermophoresis on Free Convective Heat and Mass Transfer of Viscoelastic Fluid
}

\author{
N.T.M.Eldabe, ${ }^{*}$ A.Refaie Ali, G.A.Shalaby \\ Department of Mathematics, Faculty of Education, Ain Shams University, Heliopolis, Cairo, Egypt \\ Department of Mathematics and Computer Science, Faculty of Science, Menoufia University, Egypt \\ Department of Mathematics and Computer Science, Faculty of Science, Menoufia University, Egypt \\ *ahmed.r.master@gmail.com,ahmed.refaie@science.menofia.edu.eg
}

\begin{abstract}
A theoretical study has been developed to investigate the influence of thermophoresis and couple stresses on steady flow of non-Newtonian fluid with free convective heat and mass transfer over a channel bounded by two permeable plates. The considered non-Newtonian fluid follows a viscoelastic model. The problem is modulated mathematically by a system of non-linear differential equations which pertaining to describe the continuity, momentum, energy and concentration. These equations involve the effects of viscous dissipation and chemical reaction. The numerical solutions of the dimensionless equations are found as a functions of the physical parameters of this problem. The numerical formulas of the velocity $(u)$, temperature $(\theta)$ and concentration $(\phi)$ as well as skin friction coefficient $\tau^{*}$, Nusselt number $(N u)$ and Sherwood number $(S h)$ are computed. The physical parameters effects of the problem on these formulas are described and illustrated graphically through some figures and tables. It is observed that any increase in thermophoretic parameter $\tau$ leads to reduce in velocity profiles as well as concentration layers. In contrast, the velocity increases with increasing couple stresses inverse parameter.
\end{abstract}

Keywords: Viscoelastic, Thermophoresis, Couple stresses, Heat and Mass transfer

\section{Introduction}

A transport processes of non- Newtonian fluids through a vertical channel is of great interest in diverse fields like ground-water hydrology and petroleum-reservoir. So, process of heat and mass transfer [19] in convective flows has a broad applications which actually exist not only in aeronautics and chemical process industries but also in vast industrial engineering applications and fluid fuel nuclear reactor. Most of scholars and researchers specially mathematicians, physicists, geologists and others have given the attention to these important issues especially under the influences of chemical reaction, couple stresses and thermophoresis. Influence of chemical reaction through heat and mass transfer plays the cornerstone in every chemical process and industries such as food processing and polymer production. In the light of the preceding studies concerned with flow between two vertical plates furthermore, showing important physical phenomenas such as phenomena of thermophoresis and couple stresses on non-Newtonian fluids which affect 
on flow motion; we found that Piazza [1] has presented thermophoresis definition which mean moving particles with thermal gradients, consequently particle thermophoresis is a non-equilibrium cross-flow influence between heat and mass transport. Aside from it is already exploited as a novel tool in macromolecular fractionation, micro-fluidic manipulation and selective tuning of colloidal structures. Uddin et al [2] have investigated effects of hydromagnetic and thermophoresis of unsteady forced convection boundary layer flow over flat plates. The analysis of viscoelastic fluid flow on unsteady two-dimensional in a porous channel with radiative heat transfer and mass transfer has been studied by Das [3]. Sudhakar et al [4] have studied heat and mass transfer on unsteady free convection of a Walters-B viscoelastic fluid flow past a semi infinite plate under thermophoresis effect. Recently, El-Dabe et al [5] have analyzed the effects of thermophoresis and hall currents on non-linear heat and mass transfer of second grade fluid flow over permeable infinite vertical plate with presence of viscous dissipation and chemical reaction.

Couple stresses are consequence of considering that the mechanical action of one part of a body on another, across a surface, is equivalent to a force and a moment distribution; the state of stress is measured by a stress tensor $\left(T_{i j}\right)$ and a couple stress tensor $\left(M_{i j}\right)$. El-Dabe et al [11] have discussed effect of couple stresses on the MHD of a non-Newtonian unsteady flow between two parallel porous plates by using the Eyring - Powell model. The Hall and Ion-slip effects on fully developed electrically conducting couple stress fluid flow between vertical parallel plates in the presence of a temperature dependent heat source are investigated by Srinivasacharya et al [12]. Problem of mixed convection flow of couple stress fluid between parallel vertical plates with Hall and Ion-slip effects has been indicated by Srinivasacharya et al [13]. After that, analysis of Soret and Dufour effects in the presence of chemical reaction on natural convection heat and mass transfer of a couple stress fluid in a vertical channel has been presented by Srinivasacharya et al [14]. Without a doubt to importance of couple stresses effect on viscoelastic second grade model. So to be more closer to the subject of research, El-Dabe et al [15] have studied effect of couple stresses on a pulsatile magnetohydrodynamic viscoelastic flow through a channel bounded by two permeable parallel plates. The flow of hydromagnetic non-Newtonian fluid under couple stresses through a porous channel is investigated using the Eyring-Powell model have been elucidated by Adesanya et al [16]. As can be seen more important related researches to our issue in the final analysis, Kaladhar et al. [17] have studied mixed convection flow of couple stress fluid in a vertical channel with radiation and Soret effects. Based on the above review survey, a number of questions which were overlooked in the previous papers can be arisen in our research; like what about the influences of thermophoresis with couple stresses and chemical reaction on viscoelastic second grade fluid. And also, what about the flow with these effects in viscous dissipation and nature convection heat and mass transfer .

This paper focuses to extend the work of [15, 17]. So, It is concerned with the influences of thermophoresis and couple stresses on steady flow of second grade fluid with free convective heat and mass transfer over a channel bounded by two permeable plates in the presence of viscous dissipation and chemical reaction.

\section{Materials and Methods}

Let us consider a steady free convection flow of a couple stress viscoelastic fluid [18] between two permeable vertical plates distance $2 d$ apart. By choosing the coordinate system such that $x$ - axis be taken along vertically upward direction through the central line of the channel, $y$-axis is perpendicular to the plates. The plate $y=d$ has given the constant temperature $T_{1}$ and concentration $C_{1}$, while the plate $y=d$ is subjected to a uniform temperature $T_{2}$ and concentration $C_{2}$ as shown in fig. 1. The fluid is being injected into the channel through the plate $y=-d$, and is 
being sucked through the plate $y=+d$ with uniform velocity $v_{o}$. Since the boundaries in the $x$ direction are of infinite dimensions, without loss of generality, so the physical quantities are functions in $y$ only.

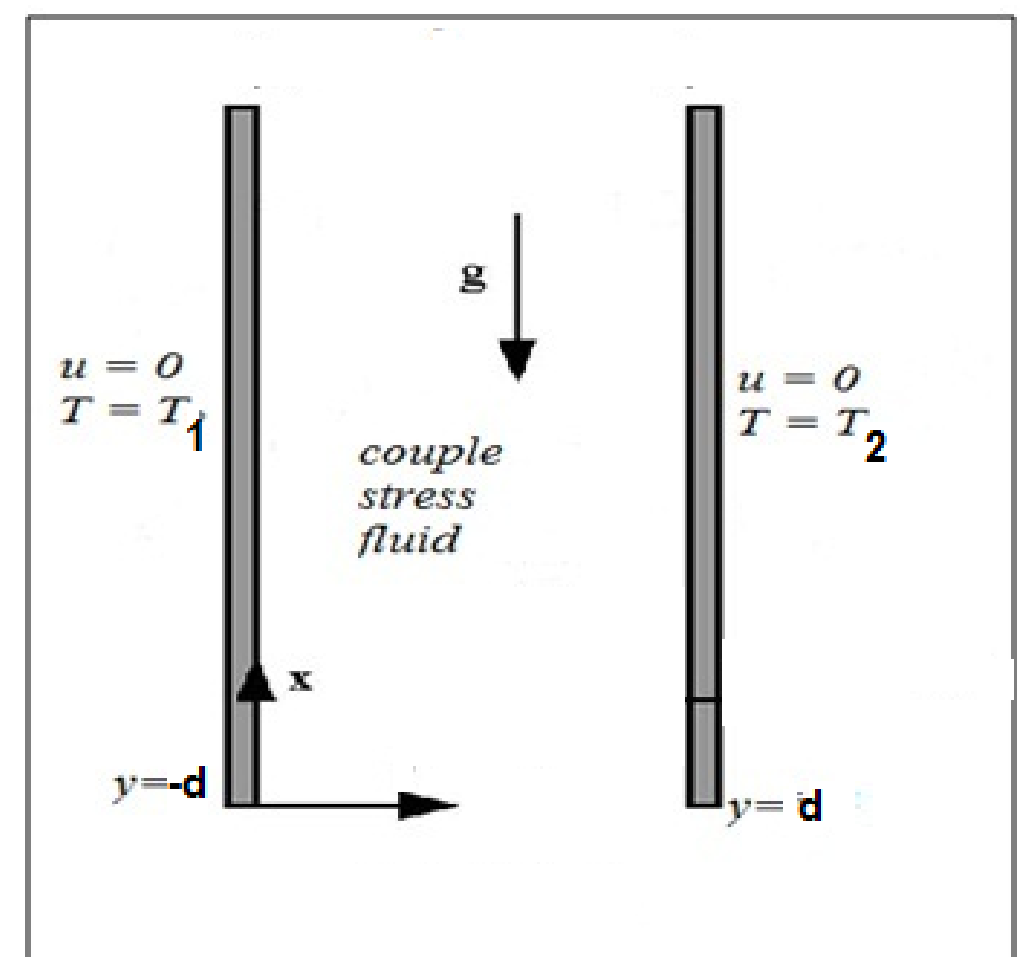

Figure 1: Coordinate system and physical configuration of present study.

The main influence of couple stresses will be to introduce a length-dependent effect that is not present in the classical non-polar theories. The governing equations of motion are [9]:

- Continuity equation

$$
V_{i, i}=0
$$

- Cauchy's first law of motion

$$
\rho a_{i}=T_{j i, j}+\rho f_{i}
$$

- Cauchy's second law of motion

$$
M_{j i, j}+\rho \ell_{i}+e_{i j k} T_{j k}=0
$$

where, $V_{i}$ is the velocity vector, $\rho$ is the density of the fluid, $a_{i}$ is the acceleration, $T_{j i}$ is the stress tensor, $f_{i}$ is the body force and $\ell_{i}$ is the body moment per unit mass. Stokes, Mindlin and Tiersten [9, 10] are obtained the constitutive equations as:

$$
\begin{gathered}
T_{i j}^{s}=-P \delta_{i j}+\alpha\left(D_{r r}\right) \delta_{i j}+2 \mu D_{i j} \\
M_{i j}^{D}=4 \eta K_{i j}+4 \eta^{\prime} K_{j i}
\end{gathered}
$$

where, $P$ is the pressure, $D_{i j}$ is the rate of deformation tensor, $\delta_{i j}$ is the Kronecker delta, $\alpha$ and $\mu$ are the material constants of viscosity, $K_{i j}$ is the curvature-twist tensor, $\eta$ and $\eta^{\prime}$ are the material constants of momentum and $M_{i j}$ is 
the couple stress tensor. The equation of motion as obtained by Stokes is:

$$
\rho a=-P_{i}+(\alpha+\mu) V_{r, r i}+\mu V_{i, r r}-\eta V_{i, t t r r}+\eta V_{r, t t r i}+\frac{1}{2} e_{i r s}\left(\rho \ell_{s}\right), r+\rho f_{i}
$$

which, in the notation of Gibbs, can be written as:

$$
\rho a=-\nabla P+(\alpha+\mu) \nabla \nabla \cdot V+\eta \nabla^{2} \nabla \nabla \cdot V+\mu \nabla^{2} V-\eta \nabla^{4} V+\frac{1}{2} \nabla \wedge(\rho \ell)+\rho f
$$

where, $a=\frac{\partial V}{\partial t}+V \cdot \nabla V$, For incompressible fluids $d i v V=0$ so, if the body force $f$ and the body moment are absent, the equations of motion reduce to

$$
\rho a=-\nabla P+\mu \nabla^{2} V-\eta \nabla^{4} V
$$

The last term in this equation gives the effect of couple stresses. For a complete description of the problem, six boundary conditions are needed for eq. 7. Three conditions are provided by assuming that the velocity of the fluid relative to the surface is zero at all solid boundaries. The remaining three conditions are provided by assuming either the couple stress is identically zero at the boundary, or that the vorticity at the boundary equal the rate of rotation of the boundary.

For the description of the thermodynamical or mechanical behavior of non-Newtonian fluids, much work has been devoted to the study of second grade fluids since they are of interest for theory and experiments. For these fluids the Cauchy stress $\mathrm{T}$ and the fluid motion are related by Truesdell and Noll (1965):

$$
T=-P I+\mu A_{1}+\alpha_{1} A_{2}+\alpha_{2} A_{1}^{2}
$$

where, $-P I$ is the indeterminate spherical stress due to the constraint of incompressibility while, $\mathrm{P}$ is the hydrostatic pressure, $\mu$ is is the dynamic viscosity, $\alpha_{1}$ and $\alpha_{2}$ are material constants and $A_{1}$ and $A_{2}$ are the kinematic Rivlin-Ericksen tensors,

$$
A_{1}=(\nabla V)+(\nabla V)^{T}, A_{2}=\frac{d A_{1}}{d t}+A_{1}(\nabla V)+(\nabla V)^{T} A_{1},
$$

here, $\mathrm{d} / \mathrm{dt}$ is the material time derivative. If the fluid of second grade modeled by eq.9 is to be compatible with thermodynamics and is to satisfy the inequality of Clausius-Duhem for all motions and the assumption that the specific Helmholtz free energy of the fluid is a minimum when it is locally at rest, then the coefficients $\mu, \alpha_{1}$ and $\alpha_{2}$ describe the viscosity, visco-elasticity and cross-viscosity respectively and must satisfy:

$$
\mu \geq 0, \quad \alpha_{1} \geq 0, \quad \alpha_{1}+\alpha_{2}=0
$$

It is necessary to be noted that when $\alpha_{1}=\alpha_{2}=0$ then, the constitutive equation of second grade fluid is respond to that of viscous fluid.

$\mathrm{Wu}$ and Greif [8] have determined the thermophoretic velocity $V_{T}$ as

$$
V_{T}=\frac{-k_{t} v}{T_{r}} \nabla T=\frac{-k_{t} v}{T_{r}} \frac{d T}{d y}
$$

where, $T_{r}$ is some reference temperature, $k_{t} v$ represents the thermophoretic diffusivity while, $k_{t}$ is coefficient of thermophoresis which ranges in value from 0.2 to 1.2 as indicated by Batchelor and Shen [7], Also by Talbot et al. [6]:

$$
k_{t}=\frac{2 C_{s}\left(\frac{\lambda_{g}}{\lambda_{p}}+C_{t} K_{n}\right)\left[K_{n}\left(C_{1}+C_{2} \exp ^{\frac{-C_{3}}{K_{n}}}\right)\right]}{2\left(1+3 C_{m} K_{n}\right)\left(C_{t} K_{n}+\frac{\lambda_{g}}{\lambda_{p}}+\frac{1}{2}\right)},
$$


where, $C_{s}, C_{t}, C_{1}, C_{2}, C_{3}, C_{m}$ are constants and $\lambda_{g}, \lambda_{p}, K_{n}$ are the thermal conductivities of the fluid and diffused particles, Knudsen number respectively. A thermophoretic parameter $\tau$ can be defined as follows;

$$
\tau=\frac{k_{t}\left(T_{2}-T_{1}\right)}{T_{r}}
$$

typical values of $\tau$ are $0.01,0.05$ and 0.1 corresponding to approximate values of $\left(T_{2}-T_{1}\right)$ equal to 3.15 and $30 k$ for a reference temperature of $T_{r}=300 k$. Within the above framework, the governing partial differential equations of the flow under the usual Boussinesq approximation are:

Continuity equation:

$$
\frac{d v^{*}}{d y^{*}}=0
$$

Momentum conservation:

$$
\rho v_{o} \frac{d u^{*}}{d y^{*}}=\mu \frac{d^{2} u^{*}}{d y^{* 2}}+\alpha_{1} v_{o} \frac{d^{3} u^{*}}{d y^{* 3}}-\eta \frac{d^{4} u^{*}}{d y^{* 4}}+\rho g \beta_{T}\left(T^{*}-T_{1}^{*}\right)+\rho g \beta_{C}\left(C^{*}-C_{1}^{*}\right),
$$

Energy equation:

$$
v_{o} \frac{d T^{*}}{d y^{*}}=\frac{\kappa}{\rho c_{p}} \frac{d^{2} T^{*}}{d y^{* 2}}+\frac{\alpha_{1}}{\rho c_{p}} v_{o} \frac{d u^{*}}{d y^{*}} \frac{d^{2} u^{*}}{d y^{* 2}}+2 \frac{\mu}{\rho c_{p}}\left(\frac{d u^{*}}{d y^{*}}\right)^{2}+\frac{\eta}{\rho c_{p}}\left(\frac{d^{2} u^{*}}{d y^{* 2}}\right)^{2},
$$

Species concentration equation

$$
v_{o} \frac{d C^{*}}{d y^{*}}=D \frac{d^{2} C^{*}}{d y^{* 2}}-\frac{d V_{T}\left(C^{*}-C_{1}^{*}\right)}{d y^{*}}-k_{c}\left(C^{*}-C_{1}^{*}\right) .
$$

The appropriate boundary conditions for the problem are:

$$
\left.\begin{array}{lc}
\text { at } \quad y^{*}=-d & u^{*}=0, u_{y y}^{*^{\prime \prime}}=0, T^{*}=T_{1}^{*}, C^{*}=C_{1}^{*} \\
\text { as } \quad y^{*}=d & u^{*}=0, u_{y y}^{*^{\prime \prime}}=0, T^{*}=T_{2}^{*}, C^{*}=C_{2}^{*}
\end{array}\right\}
$$

where, nomenclature in the above equations are: $\nu$ is the kinematic viscosity, $\rho$ is the fluid density, $g$ is the gravitational acceleration, $\beta_{T}$ and $\beta_{c}$ are thermal expansion volumetric coefficient and volumetric coefficient of concentration expansion respectively. $T^{*}$ is denote the dimensional temperature of the fluid, $C^{*}$ is the dimensional concentration of the solute [20], $c_{p}$ is the specific heat at constant pressure, $\kappa$ is the thermal conductivity of the medium and $D$ is chemical molecular diffusivity. From eq.(15), it can be seen that $v^{*}$ is either a constant or a function of time. Thus, assuming that the suction velocity to be constant. where, $v_{o}$ is a scale of mean suction velocity, Let us obtain the dimensionless equations by using the following non-dimensional variables:

$$
\left.\begin{array}{c}
u=\frac{u^{*}}{v_{o}}, y=\frac{v_{o} y^{*}}{\nu}, \\
\theta=\frac{T^{*}-T_{1}^{*}}{T_{2}^{*}-T_{1}^{*}}, \phi=\frac{C^{*}-C_{1}^{*}}{C_{2}^{*}-C_{1}^{*}}
\end{array}\right\}
$$

Using the above dimensionless quantities (20), the system of equations (16), (17) and (18) with boundary conditions (19) can be written in dimensionless form as: 


$$
\begin{gathered}
u^{(i v)}-\alpha a^{2} u^{\prime \prime \prime}-a^{2} u^{\prime \prime}+a^{2} u^{\prime}=a^{2} G_{r} \theta+a^{2} G_{m} \phi \\
-a^{2} \theta^{\prime \prime}+P r a^{2} \theta^{\prime}=P_{r} E_{c} u^{\prime \prime 2}+\alpha E_{c} a^{2} P_{r} u^{\prime} u^{\prime \prime}+2 E_{c} a^{2} P_{r} u^{\prime 2} \\
\phi^{\prime \prime}-S_{c} \phi^{\prime}=\tau\left(\phi \theta^{\prime \prime}+\phi^{\prime} \theta^{\prime}\right)+\gamma \phi
\end{gathered}
$$

with the boundary conditions :

$$
\left.\begin{array}{ll}
\text { at } \quad y=-1 & u=0, u^{\prime \prime}=0, \theta=0, \phi=0 \\
\text { at } \quad y=1 & u=0, u^{\prime \prime}=0, \theta=1, \phi=1
\end{array}\right\}
$$

The dimensionless numbers are: $\alpha=\frac{\alpha_{1} v_{o}^{2}}{\rho v^{2}}$ is the viscoelastic parameter, $\frac{1}{a^{2}}=\frac{\eta v_{o}^{2}}{\rho \nu^{3}}$ is the couple stresses parameter, $G r=\frac{g \beta_{T}\left(T_{2}^{*}-T_{1}^{*}\right) \nu}{v_{o}^{3}}$ is the Grashof number, $G m=\frac{g \beta_{c}\left(C_{2}^{*}-C_{1}^{*}\right) \nu}{v_{o}^{3}}$ is the modified Grashof number, the Prandtl number $\operatorname{Pr}=\frac{\mu c_{p}}{\kappa}$ that represents the ratio of momentum to thermal diffusivity, $E c=\frac{v_{o}^{2}}{c_{p}\left(T_{2}^{*}-T_{1}^{*}\right)}$ is the Eckert number, the Schmidt number $S c=\frac{\nu}{D}$ that represents the ratio of momentum to mass diffusivity, $\gamma=\frac{k_{c} \nu}{v_{o}^{2}}$ is the non-dimensional chemical reaction parameter, $\tau=\frac{k_{t}}{T_{r}}\left(T_{2}^{*}-T_{1}^{*}\right)$ is the thermophoretic parameter .

\section{Skin friction}

The skin friction coefficient at the surface $y=0$ is given by:

$$
\tau^{*}=-\left.\left(\frac{d u}{d y}\right)\right|_{y=0}
$$

Table 1 shows numerical values of the Skin friction coefficient $\tau^{*}$ for various values of viscoelastic parameter $\alpha$, couple stress inverse parameter $a$, Grashof number $G r$, modified Grashof number Gm, Prandtl number Pr, Schmidt number $S c$ and thermophoresis parameter $\tau$. From that table, we noticed that an increase in $P r, S c$ and $\tau$ increases the value of the skin friction coefficient $\tau^{*}$, while an increase in $\alpha, a, G r$ and $G m$ decreases the value of the skin friction coefficient $\tau^{*}$.

\section{Nusselt Number}

From the temperature field; the rate of heat transfer between plates and the fluid can be expressed in terms of non-dimensional Nusselt number as $q_{w}=-\kappa \frac{d \theta}{d y}=\kappa N u$ where the Nusselt number is given by:

$$
N u=-\left.\left(\frac{d \theta}{d y}\right)\right|_{y=0}
$$

Nusselt number $N u$ is given in table2. It can be clearly seen that an increase in the Prandtl number $\operatorname{Pr}$ result in an increase in Nusselt number $N u$. Because of the increase in viscoelastic parameter $\alpha$, couple stress inverse parameter $a$, Grashof number $G r$ and Eckert number Ec then Nusselt number $N u$ decreases. 
Table 1: Skin friction coefficient $\tau^{*}$ data for: $\alpha=0.2, a=0.5 G r=5, G m=5, \operatorname{Pr}=0.71, E c=0.01, S c=0.22$, $\tau=0.2, \gamma=0.3$

\begin{tabular}{cccccccc}
\hline$\alpha$ & $a$ & $G r$ & $G m$ & $\operatorname{Pr}$ & $S c$ & $\tau$ & $\tau^{*}$ \\
\hline 0.2 & 0.5 & 5 & 5 & 0.71 & 0.22 & 0.2 & -0.0251 \\
5 & 0.5 & 5 & 5 & 0.71 & 0.22 & 0.2 & -0.0379 \\
0.2 & 0.1 & 5 & 5 & 0.71 & 0.22 & 0.2 & -0.0009 \\
0.2 & 0.4 & 5 & 5 & 0.71 & 0.22 & 0.2 & -0.0157 \\
0.2 & 1 & 5 & 5 & 0.71 & 0.22 & 0.2 & -0.1125 \\
0.2 & 0.5 & 0.2 & 5 & 0.71 & 0.22 & 0.2 & -0.0131 \\
0.2 & 0.5 & 10 & 5 & 0.71 & 0.22 & 0.2 & -0.0376 \\
0.2 & 0.5 & 5 & 0.2 & 0.71 & 0.22 & 0.2 & -0.0130 \\
0.2 & 0.5 & 5 & 15 & 0.71 & 0.22 & 0.2 & -0.0505 \\
0.2 & 0.5 & 5 & 5 & 0.9 & 0.22 & 0.2 & -0.0246 \\
0.2 & 0.5 & 5 & 5 & 1.7 & 0.22 & 0.2 & -0.0223 \\
0.2 & 0.5 & 5 & 5 & 0.71 & 0.60 & 0.2 & -0.0243 \\
0.2 & 0.5 & 5 & 5 & 0.71 & 0.96 & 0.2 & -0.0232 \\
0.2 & 0.5 & 5 & 5 & 0.71 & 0.22 & 0.1 & -0.0252 \\
0.2 & 0.5 & 5 & 5 & 0.71 & 0.22 & 0.9 & -0.0248 \\
\hline
\end{tabular}

Table 2: Nusselt number $N u$ data for: $\alpha=0.2, a=0.5 G r=5, G m=5, \operatorname{Pr}=0.71, E c=0.01, S c=0.22, \tau=0.2$, $\gamma=0.3$

\begin{tabular}{cccccc}
\hline$\alpha$ & $a$ & $G r$ & $\operatorname{Pr}$ & $E c$ & $N u$ \\
\hline 0.2 & 0.5 & 5 & 0.71 & 0.01 & -0.4613 \\
5 & 0.5 & 5 & 0.71 & 0.01 & -0.4617 \\
0.2 & 0.1 & 5 & 0.71 & 0.01 & -0.4604 \\
0.2 & 0.8 & 5 & 0.71 & 0.01 & -0.4626 \\
0.2 & 0.5 & 0 & 0.71 & 0.01 & -0.4606 \\
0.2 & 0.5 & 20 & 0.71 & 0.01 & -0.4664 \\
0.2 & 0.5 & 5 & 0.9 & 0.01 & -0.4397 \\
0.2 & 0.5 & 5 & 1.7 & 0.01 & -0.3234 \\
0.2 & 0.5 & 5 & 0.71 & 0.09 & -0.4695 \\
0.2 & 0.5 & 5 & 0.71 & 0.6 & -0.5386 \\
\hline
\end{tabular}

\section{Sherwood Number}

From the concentration field; the rate of mass transfer between plates and the fluid can be expressed in terms of non-dimensional Sherwood number as $j_{m}=-\rho D \frac{d \phi}{d y}=\rho D S h$ where the Sherwood number is given by:

$$
S h=-\left.\left(\frac{d \phi}{d y}\right)\right|_{y=0}
$$

Table 3 shows numerical values of mass transfer coefficient in terms of sherwood number $S h$. It is observed that Sherwood number increases with increasing in Prandtl parameter $P r$, Schmidt number $S c$, thermophoretic parameter $\tau$ and chemical reaction parameter $\gamma$. likewise, the increase of Eckert number Ec leads to decrease the Sherwood number. 
Table 3: Sherwood number $S h$ data for $\alpha=0.2, a=0.5 G r=5, G m=5, \operatorname{Pr}=0.71, E c=0.01, S c=0.22, \tau=0.2$, $\gamma=0.3$

\begin{tabular}{cccccc}
\hline$P r$ & $E c$ & $S c$ & $\tau$ & $\gamma$ & $S h$ \\
\hline 0.71 & 0.01 & 0.22 & 0.2 & 0.3 & -0.4636 \\
1.7 & 0.01 & 0.22 & 0.2 & 0.3 & -0.4585 \\
0.71 & 0.09 & 0.22 & 0.2 & 0.3 & -0.4640 \\
0.71 & 0.9 & 0.22 & 0.2 & 0.3 & -0.4707 \\
0.71 & 2 & 0.22 & 0.2 & 0.3 & -0.4809 \\
0.71 & 0.01 & 0.60 & 0.2 & 0.3 & -0.4254 \\
0.71 & 0.01 & 0.78 & 0.2 & 0.3 & -0.4015 \\
0.71 & 0.01 & 0.96 & 0.2 & 0.3 & -0.3751 \\
0.71 & 0.01 & 0.22 & 0.1 & 0.3 & -0.4657 \\
0.71 & 0.01 & 0.22 & 0.6 & 0.3 & -0.4548 \\
0.71 & 0.01 & 0.22 & 0.9 & 0.3 & -0.4480 \\
0.71 & 0.01 & 0.22 & 0.2 & 0.1 & -0.4820 \\
0.71 & 0.01 & 0.22 & 0.2 & 0.5 & -0.4464 \\
0.71 & 0.01 & 0.22 & 0.2 & 0.8 & -0.4227 \\
\hline
\end{tabular}

\section{Results and Discussion}

In order to investigate the physical significance of the problem, a program was designed by using Mathematica 10 software including using of parametric ND solve package to simulate the numerical solutions of the system of the differential equations which describe our problem. The purpose of these numerical computations to illustrate the influence of various governing physical parameters such as: viscoelastic parameter $\alpha$, couple stress inverse parameter $a$, Grashof number $G r$, modified Grashof number $G m$, Prandtl number Pr, Eckert number Ec, Schmidt number $S c$, thermophoretic parameter $\tau$ and dimensionless chemical reaction parameter $\gamma$ on the velocity $u(y)$, the temperature $\theta(y)$ and the concentration $\phi(y)$ fields which they have been done at the following values: $\alpha=0.2, a=0.5 G r=5$, $G m=5, \operatorname{Pr}=0.71, E c=0.01, S c=0.22, \tau=0.2, \gamma=0.3$. As well as, Skin friction $\tau^{*}$, Nusselt number $N u$ and Sherwood number $S h$ have been computed and represented in tabulated forms at same values of parameters. In order to get clear insight into the physics of the problem, a representative set of numerical results are shown graphically in figures $(2-19)$. In fig. 2 , by increasing the viscoelastic second grade parameter $\alpha$, the velocity profile $u(y)$ decreases. Since the influence of $\alpha$ on the temperature $\theta(y)$ and concentration $\phi(y)$ fields is very small; then the results are not shown here. It is observed from fig. 3 that an increase in couple stress inverse parameter $a$ leads to a large arise in the values of velocity $u(y)$. The velocity $u(y)$ curves decrease when the thermophoretic parameter $\tau$ increases as seen in fig. 4. The values of Grashof number $G r$ are taken to be positive and negative as they respectively represent symmetric heating of the plates when $G r<0$ and symmetric cooling of the plates when $G r>0$. The velocity profiles $u(y)$ increases not only by increasing the Grashof number $G r$ but also by increasing the modified Grashof number $G m$ as shown in fig. 5 and fig. 6 respectively. This is due to the buoyancy forces which is acting on the fluid particles due to gravitational force that enhances the fluid velocity. The increase in the Prandtl number $\operatorname{Pr}$ results in the decrease in $u(y)$ as displayed in fig. 7. This is physically true because, the Prandtl number is a non-dimensional number which is the ratio of momentum diffusivity (kinematic viscosity) to thermal diffusivity. The effect of increasing Eckert number $E c$ is to enhance $u(y)$ as shown in fig. 8. Higher Eckert number values lead to greater viscous dissipative heat. On the 
contrary, the reversed effect has been observed where fig. 9 indicates that velocity curves decreases with increasing of Schmidt number Sc. Physically it is true as the concentration increase the density of the fluid increases which results a decrease in fluid particles. fig. 10 depicts that $u(y)$ decreases as the chemical reaction parameter $\gamma$ increases because of the presence of viscous dissipation. As a general description, velocity curves of this fluid are increased from injected plate to reach the top of the curves (the highest value for the speed) which locate next to the center of channel and then they decrease until it reaches zero at the sucked plate. This mean that the velocity profiles of the fluid is maximum at the center of the channel and zero at the plates.

\section{velocity profiles}

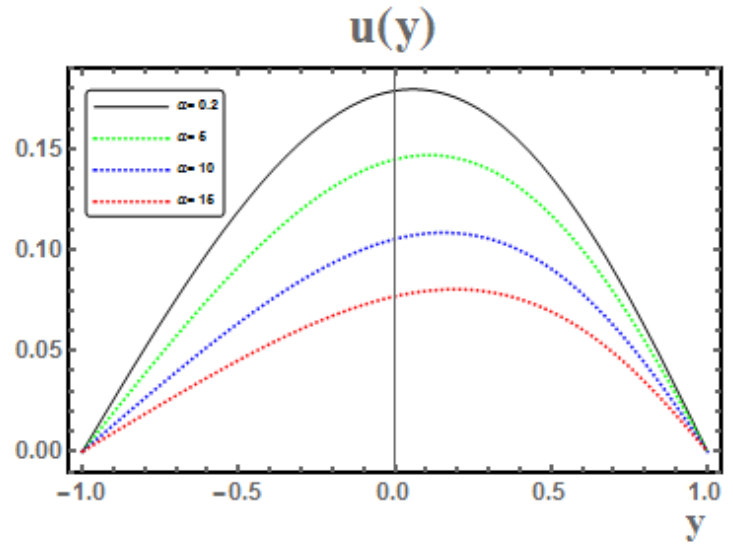

Figure 2: Effect of the viscoelastic parameter $\alpha$ on velocity profiles $u(y)$.

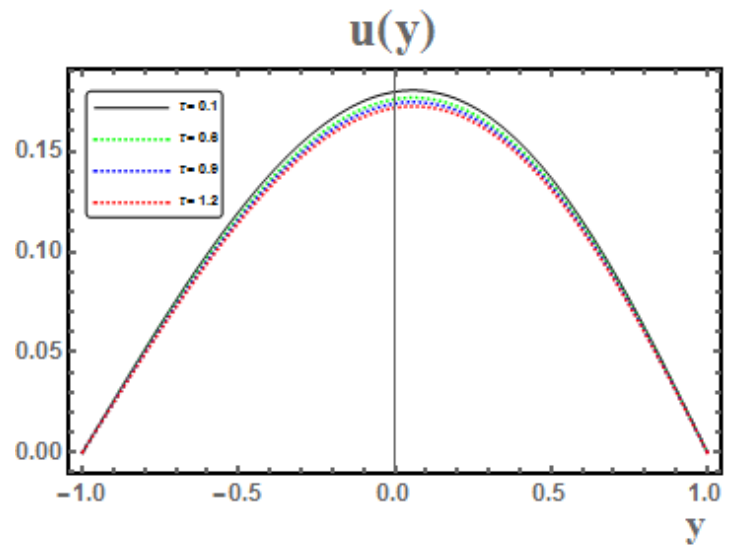

Figure 4: Effect of thermophoretic parameter $\tau$ on velocity profiles $u(y)$.

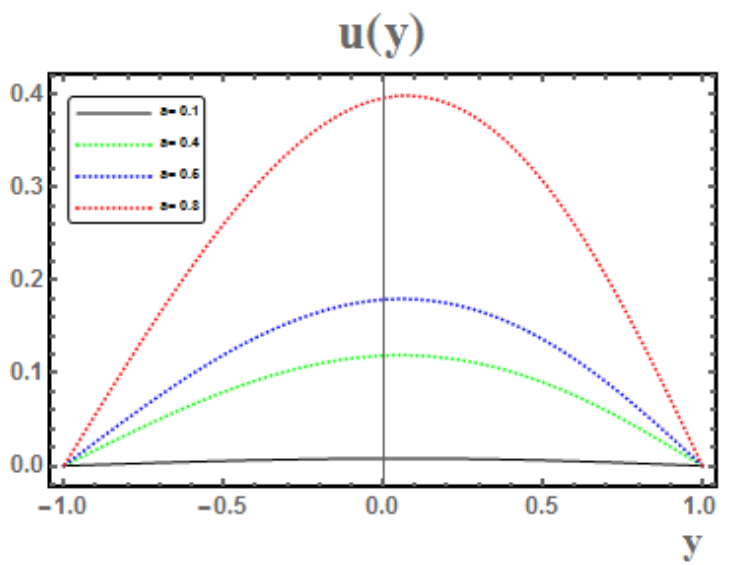

Figure 3: Effect of couple stress inverse parameter $a$ on velocity profiles $u(y)$.

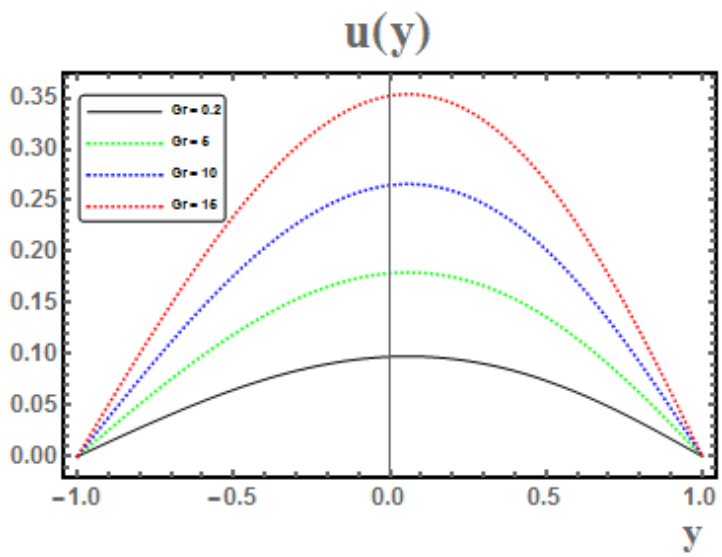

Figure 5: Effect of Grashof number $G r$ on velocity profiles $u(y)$. 


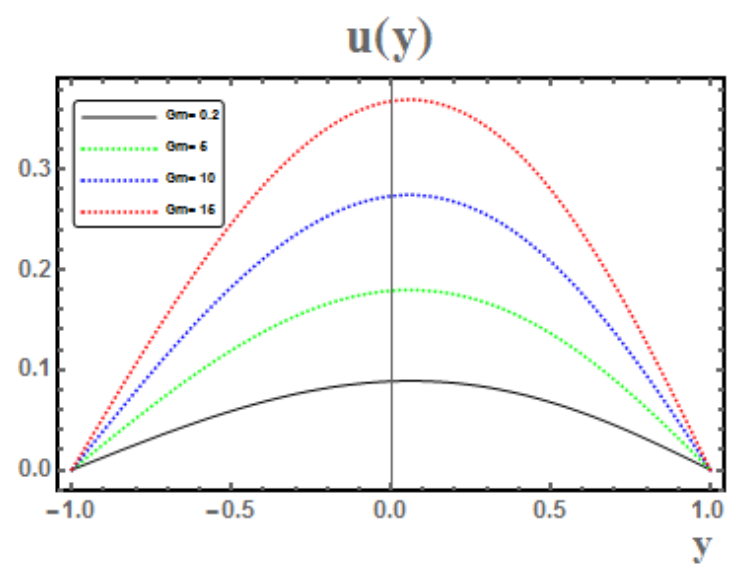

Figure 6: Effect of modified Grashof number $G m$ on velocity profiles $u(y)$.

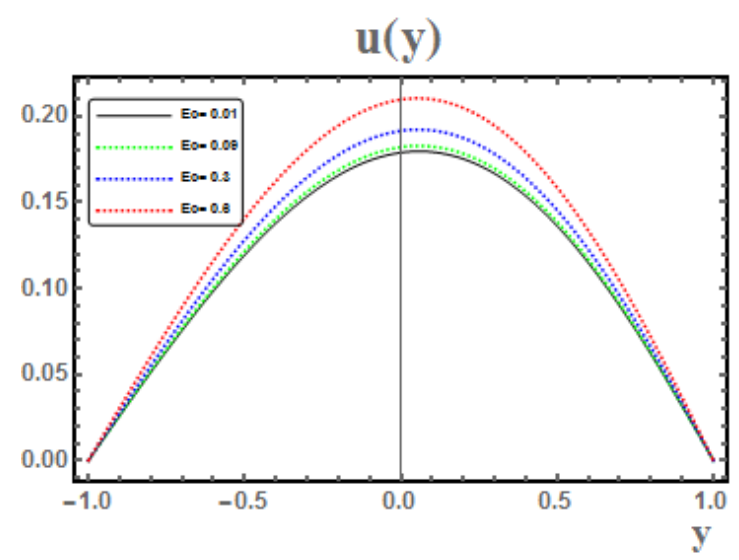

Figure 8: Effect of Eckert parameter $E c$ on velocity profiles $u(y)$.

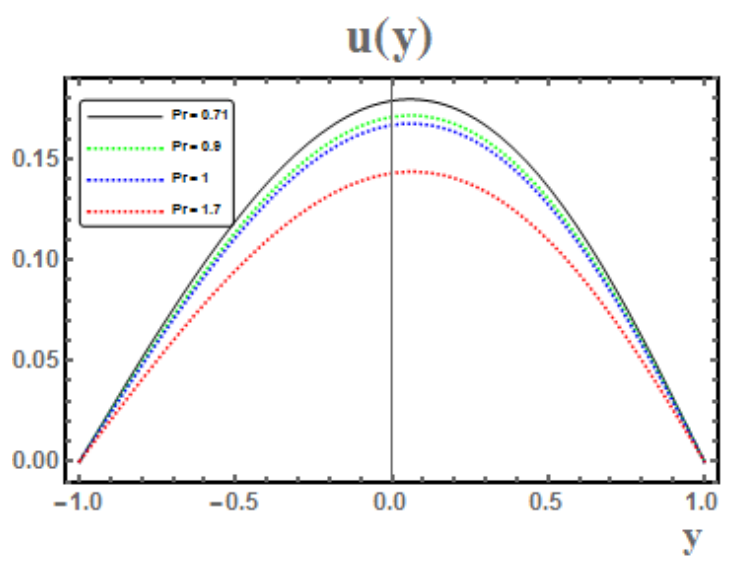

Figure 7: Influence of Prandtl parameter $P r$ on velocity profiles $u(y)$.

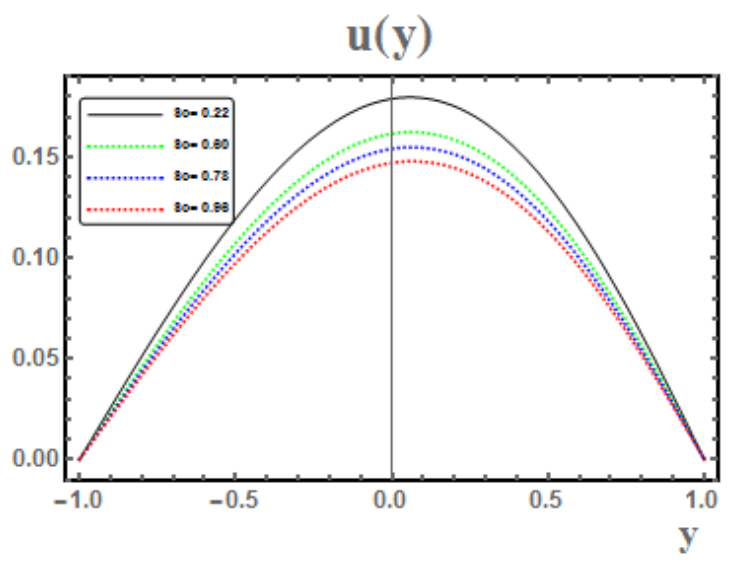

Figure 9: Effect of Schmidt parameter $S c$ on velocity profiles $u(y)$.

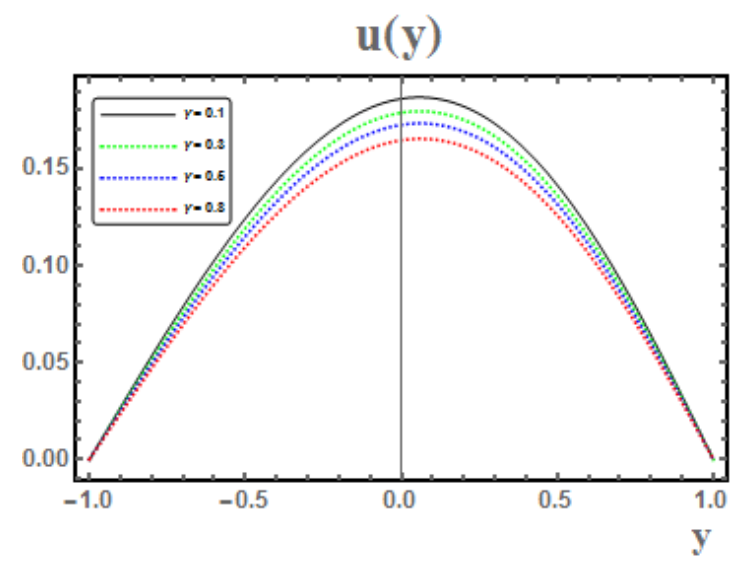

Figure 10: Effect of chemical reaction parameter $\gamma$ on velocity profiles $u(y)$. 


\section{Temperature profiles}

Fig. 11 exhibits the temperature profiles $\theta(y)$ for various values of Prandtl number $P r$. From this figure it is noticed that by increasing $\operatorname{Pr}$, the temperature decreases. Small values of the Prandtl number, $\operatorname{Pr} \ll 1$, means that the thermal diffusivity dominates. Whereas with large values of $\operatorname{Pr} \gg 1$, the momentum diffusivity dominates the behavior. By choose the values of Prandtl number where they are physically correspond to gases and fluids which are commonly used in commerce and industry. Around 0.16-0.7 for mixtures of noble gases or noble gases with hydrogen and at room temperature $\operatorname{Pr}$ is 0.71 . fig. 12 displays influence of Eckert parameter $E c$ on the temperature profiles $\theta(y)$. It is observed that temperature $\theta(y)$ increases as $E c$ increases. Effect of Grashof number $G r$ and modified Grashof number $G m$ on temperature profiles $\theta(y)$ were studied into figure 13 and 14 respectively . From the above figures it is noticed that the temperature distribution $\theta(y)$ of the fluid flow increases as increase both of $G r$ and $G m$. From these figures it is noticed that thermal boundary layer stretch when the values of $E c, G r$ and $G m$ parameters increase. Generally, The fluid has a high temperature at the positive side of the channel compared to the another part which it locates near to the injection process.

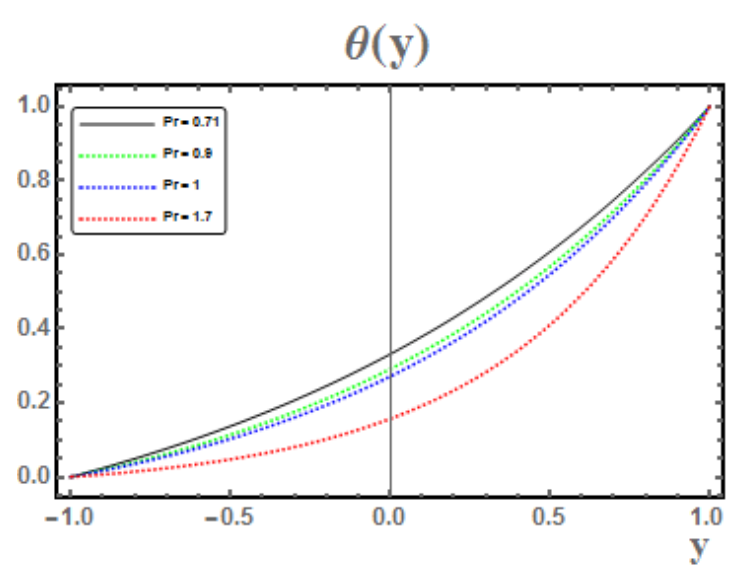

Figure 11: Influence of Prandtl parameter $\mathrm{Pr}$ on temperature profiles $\theta(y)$.

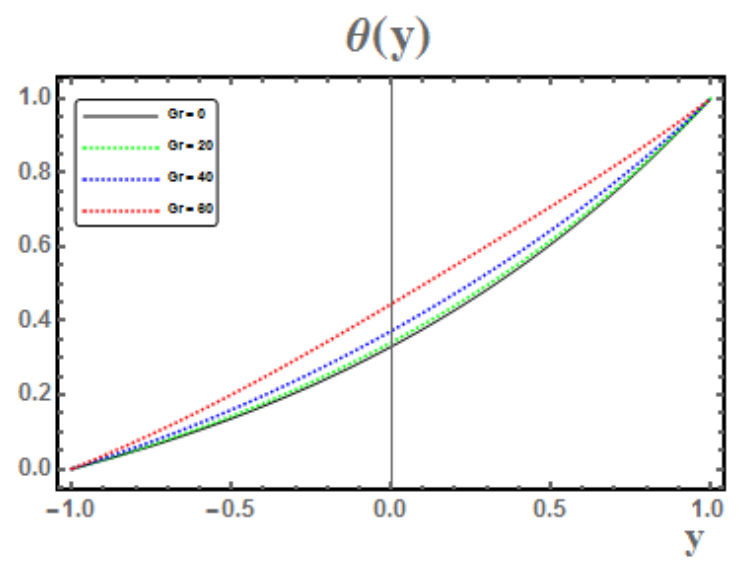

Figure 13: Influence of Grashof number $G r$ on temperature profiles $\theta(y)$.

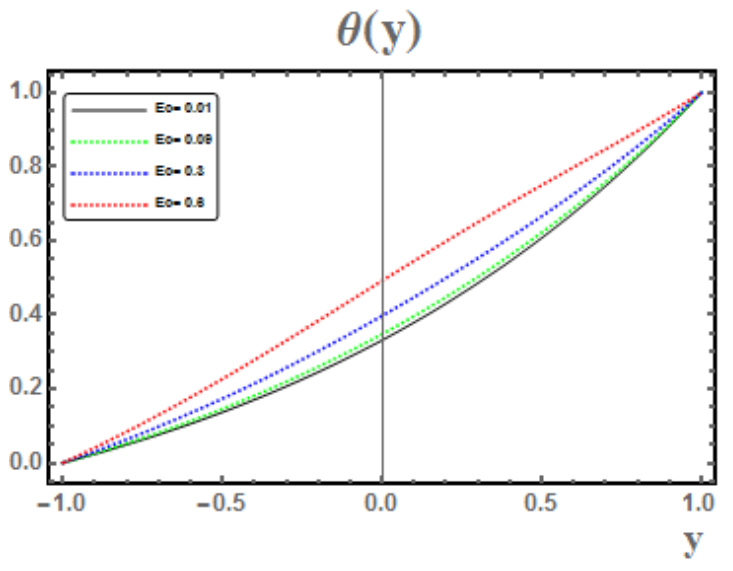

Figure 12: Influence of Eckert parameter $E c$ on temperature profiles $\theta(y)$.

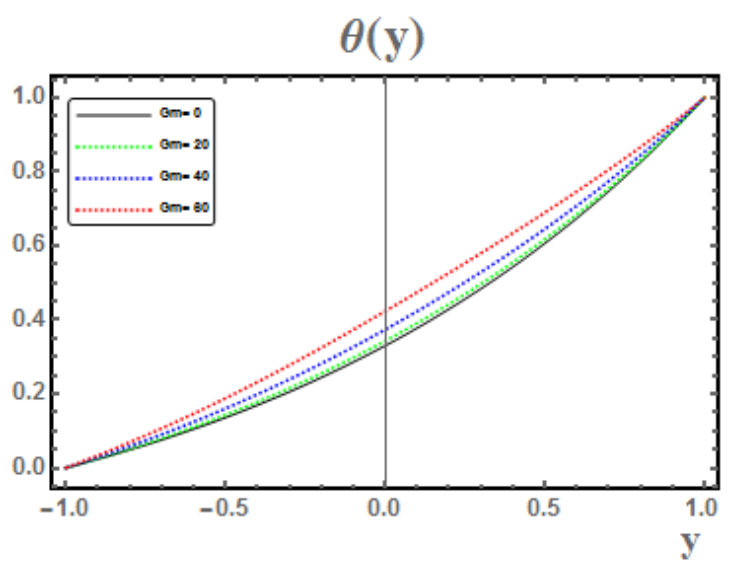

Figure 14: Influence of modified Grashof number $G m$ on temperature profiles $\theta(y)$. 


\section{Concentration profiles}

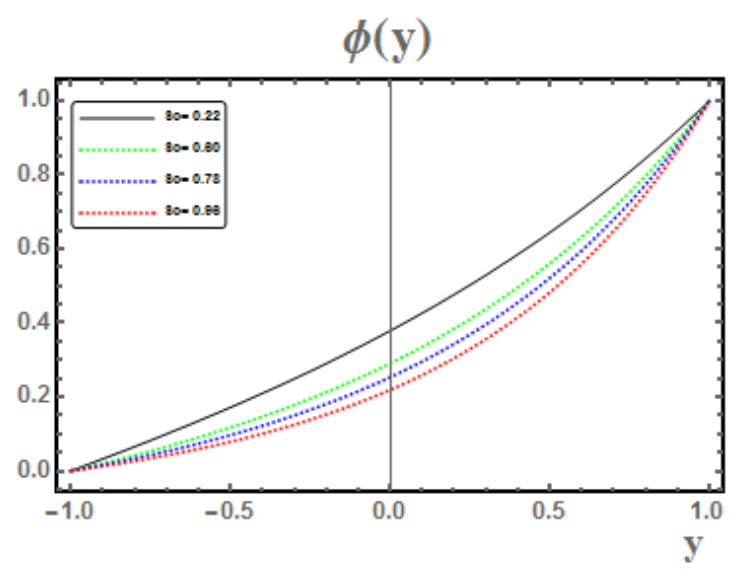

Figure 15: Effect of Schmidt parameter $S c$ on concentration profiles $\phi(y)$.

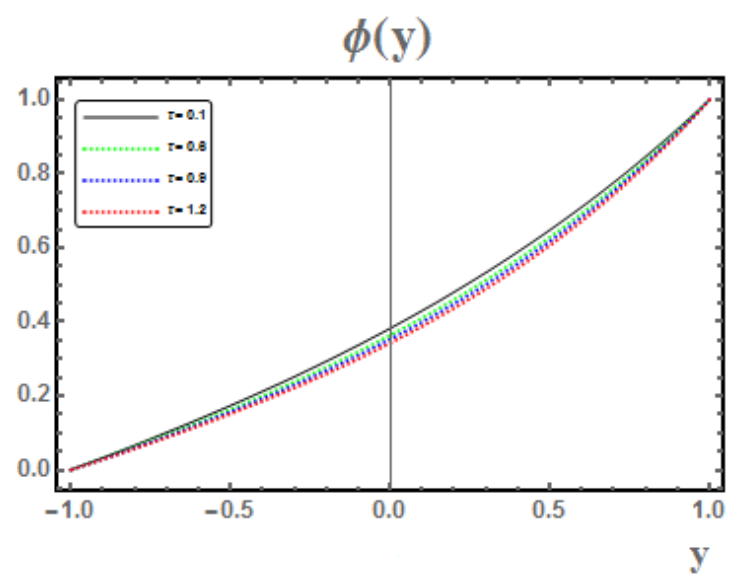

Figure 17: Influence of thermophoretic parameter $\tau$ on concentration profiles $\phi(y)$

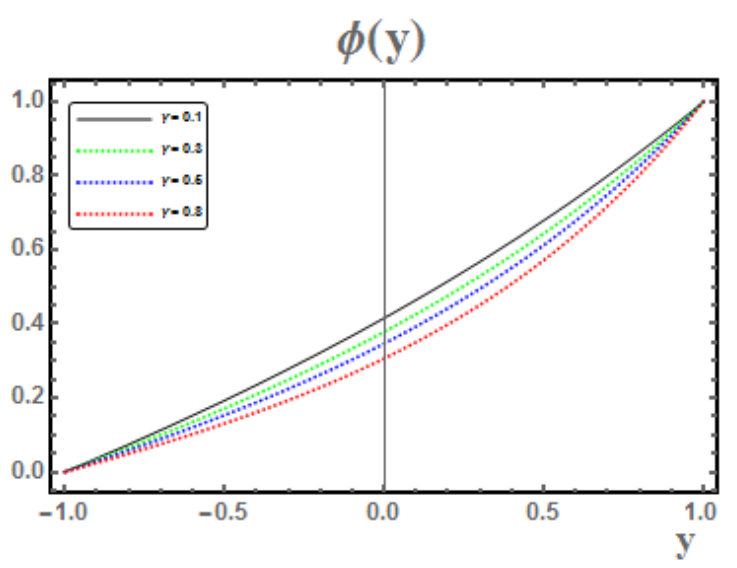

Figure 16: Influence of chemical reaction parameter $\gamma$ on concentration profiles $\phi(y)$.

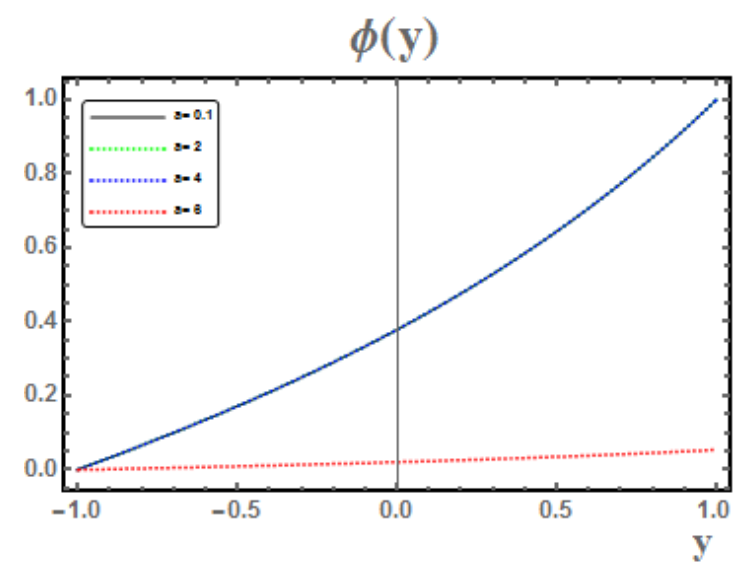

Figure 18: Effect of couple stress inverse parameter $a$ on concentration profiles $\phi(y)$.

Influence of Schmidt number $S c$ on concentration $\phi(y)$ is shown in figure 14, from this figure it is noticed that concentration decreases with an increase in Sc. Because, Schmidt number is a dimensionless number determined by the ratio of momentum diffusivity and mass diffusivity, and is used to characterize fluid flows in which there are simultaneous momentum and mass diffusion convection processes. Therefore concentration boundary layer decreases with an increase in Schmidt number. The increase in chemical reaction parameter $\gamma$ at values $\gamma=0.1,0.3,0.5,0.8$ leads to a decrease in concentration distributions $\phi(y)$ as shown in fig. 15. Effect of thermophoresis $\tau$ on concentration profiles $\phi(y)$ is displayed in fig.16 at values of $\tau=0.1,0.6,0.9$ and 1.2 . It is noticed that the concentration profiles $\phi(y)$ decreases as $\tau$ increases. Specially, the effect of increasing the thermophoretic parameter $\tau$ is limited to increasing the wall slope of the concentration profiles, but decreasing the concentration. This is true only for small values of Schmidt number for which the Sherwood number $S h$ is large as chemical reaction effect increases and compared to the convection effect. So, the thermophoretic parameter $\tau$ is expected to alter the concentration boundary layer significantly. The concentration profiles diverge at values of couple stress inverse parameter that equal to $a=8$. As a result of the effect 
of the previous parameters on the concentration layers, we found that the fluid be more concentrated in the adjacent side of the absorption process of th plate which located at $y=1$.

\section{Conflicts of Interest}

The authors declare that they have no competing interests.

\section{Funding Statement}

Funding NOT applicable.

\section{References}

[1] R. Piazza. Thermophoresis : moving particles with thermal gradients. Soft Matter - Royal Society of Chemistry, 4, pages $1740-1744,2008$.

[2] Jashim Uddin and Yeakub Ali. Effects of hydromagnetic and thermophoresis of unsteady forced convection boundary layer flow over flat plates. Journal of Applied Mathematics and Physics, 4, pages 1756-1776, 2016.

[3] U. J. Das. Viscoelastic effects on unsteady two-dimensional and mass transfer of a viscoelastic fluid in a porous channel with radiative heat transfer. Engineering, 5, pages $67-72,2013$.

[4] C. Sudhakar, N. B. Reddy, B. Vasu and V. R. Prasad. Thermophoresis effect on unsteady free convection heat and mass transfer in a Walters-B fluid past a semi infinite plate. International Journal of Engineering Research and Applications, 2, pages $2080-2095,2012$.

[5] Nabil T. M. El-dabe, A. Refaie Ali, A. A. El-shekhipy and G. A. Shalaby. Non-linear heat and mass transfer of second grade fluid flow with hall currents and thermophoresis Effects. Appl. Math. Inf. Sci., 1(11) pages 267-280, 2017.

[6] L. Talbot, R.K. Cheng, R.W. Schefer and D.R. Willis. Thermophoresis of particles in a heated boundary layer. J. Fluid Mech., 101, pages $737-758,1980$.

[7] G.K. Batchelor and C. Shen. Thermophoretic deposition of particles in gas flowing over cold surfaces. J Colloid Interface Sci, 107, pages $21-37,1985$.

[8] C.K. Wu and R. Greif. Thermophoretic deposition including an application to the outside vapor deposition process. Int. J. Heat. Mass. Tran., 39(7), pages $1429-1438,1996$.

[9] Stokes, VK. Theories of fluids with mictostructure. Springer Verlag, Berlin, 1984.

[10] Mindlin and Tiersten. HF: Arch. Ration. Mech. Anal., 11, 415, 1962. 
[11] N. T. M. Eldabe, A. A. Hassan, and Mona A. A. Mohamed. Effect of couple stresses on the MHD of a non-Newtonian unsteady flow between two parallel porous plates. Z. Naturforsch. 58a), pages $204-210,2003$.

[12] D. Srinivasacharya and K. Kaladhar. Natural convection flow of a couple stress fluid between two vertical parallel plates with Hall and Ion-slip effects. Acta Mech. Sin., 28(1), pages 41 -50, 2012.

[13] D. Srinivasacharya and K. Kaladhar. Mixed convection flow of couple stress fluid between parallel vertical plates with Hall and Ion-slip effects. Commun Nonlinear Sci Numer Simulat, 17, pages 2447-2462, 2012.

[14] D. Srinivasacharya and K. Kaladhar. Soret and Dufour effects on free convection flow of a couple stress fluid in a vertical channel with chemical reaction. Chemical Industry and Chemical Engineering Quarterly , 19 (1), pages $45-55,2013$.

[15] N.T.M. Eldabe, A.A. Hassan and Mona A.A. Mohamed. Effect of couple stresses on a pulsatile magnetohydrodynamic viscoelastic flow through a channel bounded by two permeable parallel plates. Mechanics and Mechanical Engineering, 1(7), pages 5-14, 2004.

[16] Samuel O. Adesanya, John A. Falade and Randolph Rach. Effect of couple stresses on Eyring-Powell fluid flow through a porous channel. Theoretical and applied mechanics , 42, Issue 2, pages 135-150, 2015.

[17] K. Kaladhar, S. S. Motsa and D. Srinivasacharya. Mixed convection flow of couple stress fluid in a vertical channel with radiation and soret effects. Journal of Applied Fluid Mechanics, 9, No. 1, pages 43-50, 2016.

[18] Mohyuddin, Muhammad and Thomas Goetz. Resonance behavior of viscoelastic fluid in Poiseuille flow in the presence of a transversal magnetic field. International Journal for numerical methods in fluids, 49(8), pages 837-847, 2005 .

[19] S.Asghara, Muhammad R.Mohyuddinb and T.Hayat. Effects of Hall current and heat transfer on flow due to a pull of eccentric rotating disks. International Journal of Heat and Mass Transfer, 48, pages 599-607, 2005.

[20] Javed I. Siddique, Forrest A. Landis and Muhammad R. Mohyuddin. Dynamics of Drainage of Power-Law Liquid into a Deformable Porous Material. Open Journal of Fluid Dynamics, 4, pages 403-414, 2014. 